and CT scan findings. after combined therapy, $80 \%$ of children with delayed speech delay have very good response and improvement, $77 \%$ of children with delayed milestone and hypertonia, and $87 \%$ with delayed walking have positive clinical outcomes. Both sexes have equal response to combined therapy. Such findings were obtained as a result of early treatment and diagnosis of children with (CP). In addition, improvement in CT scan results was obtained. $84 \%$ of treated children have great improvement in their neuroimaging results from moderate/severe forms of brain atrophy to mild form of brain atrophy after being treated and followed up for 6 months- 1 year.

Conclusions The damaged brain sites based on CT scan results, showed progressive improvement in response to B12 and omega-3 fatty acids upon daily supplement throughout 6 months to one year. B12 vitamin and omega- 3 fatty acids are valuable therapy for children with various forms of cerebral palsy particularly when being linked. The greatest improvement in speech and motor development was significantly observed in about 32 patients (80\%) of treated children with combined therapy. Others have less response to combine therapy as being presented and diagnosed beyond 1 year of age $(16 \%)$

\section{P517 METABOLIC FEATURES OF ADOLESCENTS WITH OBESITY}

Lyubov Rychkova*, Anna Pogodina, Lyudmila Grebenkina, Olga Berdina, Zhanna Ayurova, Tatyana Astakhova. Scientific Centre for Family Health and Human Reproduction Problems, Irkutsk, Russian Federation

\subsection{6/archdischild-2019-epa.852}

Introduction Over the last decades researchers discuss the interconnection between obesity and metabolic syndrome, the main manifestations of which include obesity, dyslipidemia, insulin resistance, and hypertension.

Aim To determine biochemical parameters in obese adolescents of Asian and Caucasian ethnicities, living in a rural area.

Materials and methods We investigated 200 adolescents with overweight and obesity aged 11-17 years: 43 Asian adolescents and 29 Caucasian adolescents. The control group comprised 128 adolescents with normal body weight in both ethnic groups. All adolescents were living in Buryatia (Russia). Overweight was diagnosed when body mass index (BMI) $>85$ percentile, obesity - at BMI $\geq 95$. Metabolic status was established by the spectrophotometric method. The following parameters were determined and analyzed: total cholesterol, high and low density lipoproteins fractions, triglycerides, fasting glucose and two-hour post-load glucose levels.

Results After comparison of biochemical parameters, we found changes in carbohydrate metabolism in obese adolescents. Glucose tolerance test showed significant differences between twohour post-load glucose levels between groups with obesity and normal body weight $(4.63 \pm 0.62 \mathrm{mmol} / \mathrm{L}$ against $3.89 \pm$ $0.62 \mathrm{mmol} / \mathrm{L}$, respectively in Asian adolescents, $\mathrm{p}=0.000$ $4.48 \pm 0.9 \mathrm{mmol} / \mathrm{L}$ against $3.89 \pm 0.5 \mathrm{mmol} / \mathrm{L}$, respectively in Caucasian adolescents, $p=0.000$ ). We found significant differences between levels of this parameter among adolescents with obesity of Asian and Caucasian ethnicities (4.63 \pm 0.62 $\mathrm{mmol} / \mathrm{L}$ against $4.48 \pm 0.9 \mathrm{mmol} / \mathrm{L}$, respectively, $\mathrm{p}=0.006)$. Mean group values of fat metabolism in adolescents with obesity were within normal values, however during a frequency analysis we detected changes: hypercholesteremia in $6.8 \%$ in
Asian adolescents and 4.6\% in Caucasian adolescents. Hypertriglyceridemia was detected in $16.3 \%$ cases of Asians and $9.2 \%$ in the Caucasian group, $\mathrm{p}=0.007$. Hypolipoproteinemia in obese adolescents was noticed in $6.7 \%$ of Asians (in Caucasian group $-3.4 \%, \mathrm{p}=0.569$ ).

Conclusions Therefore all metabolic changes, mentioned above, are more typical for the natives than for newcomer, namely, Caucasian people, which probably may be associated with food stereotypes and consuming food rich in animal fats. At the same time traditional food stereotypes in Asian ethnicities may contribute to adaptation to atherogenesis and development of resistance to atherosclerosis and diabetes mellitus, predominantly of the type 1 .

\section{P518 FUNCTIONAL DISORDERS OF THE COLON IN ADOLESCENTS WITH OBESITY: PSYCHOLOGICAL CHARACTERISTICS}

Anastasiya Romanitsa, Lyubov Rychkova*, Anna Pogodina. Scientific Centre for Family Health and Human Reproduction Problems, Irkutsk, Russian Federation

\subsection{6/archdischild-2019-epa.853}

Adolescent obesity is a very serious problem of the 21 century. Approximately $86.5 \%$ of obese adolescents have functional disorders of the colon (FDC), among which the most prevalent disorder is irritable bowel syndrome (IBS) - 76.1\%. One of the causes of FDC is a psychological state. Psychological problems, may develop into more serious conditions, such as severe depression, low self-esteem, anorexia nervosa, and bulimia nervosa.

Aim To determine psycho-emotional disorders in adolescents with obesity and FDC.

Materials and methods We examined 111 children with obesity (11-17 years), among them 64 girls and 47 boys, age 14.5 \pm 2.0 , over a period 2016-2018. Depending on the presence of FDC in adolescents with obesity, the sample was divided in 3 groups: 1 group included $73(65.8 \%)$ adolescents with obesity and IBS (diagnosed according to The Rome IV criteria), 2 group - $23(20.7 \%)$ adolescents with obesity and other FDC, 3 group - 15 (13.5\%) adolescents with obesity without FDC. We conducted a questionnaire survey on the psychoemotional state of adolescents (State-Trait Anxiety Inventory, Rosenberg's Faith in People scale, Beck Depression Inventory, EAT-26). Results were processed using software Statistica 10.0 for Windows. The difference was considered significant at $\mathrm{p}<0.05$. Results EAT-26 did not show differences between groups on the following indicators: pursuance of leanness, bulimia, inefficiency, interoceptive incapacity. There were significant differences in the group with IBS $(p=0.000)$ on discomfort with one's body, distrust in interpersonal relationships. Rosenberg's Faith in People scale did not show differences between groups $(p=1.2)$. State-Trait Anxiety Inventory showed that state anxiety and trait anxiety are more pronounced in groups with IBS and other FDC. There is a significant difference between groups with IBS and other FDC and the group without FDC $(p=0.000)$. Depression is significantly higher in the group with IBS $(p<0.001)$ according to Beck Depression Inventory.

Conclusions We found maladaptive eating behavior (pursuance for leanness, bulimia, inefficiency, interoceptive incapability) in adolescents with obesity, regardless of FDC. We observed increased discomfort with the body, distrust in interpersonal 